\title{
SYNTHESIS OF 2-(1,3-DIARYPYRAZOL-5-YLAMINO)- 4(3-OXO-2H-1,4-BENZOXA/THIAZIN-6-YL)-THIAZOLES
}

\author{
K. N. Jayaveera and S. Sailaja \\ Oil Technological Research Institute, Jawaharlal Nehru Technological University, \\ Anantapur - 515001 and \\ G. Jagath Reddy* and K. Srinivasa Rao
}

R \& D Laboratories, Dr. Jagath Reddy's Heterocyclics, 81, S.V.Co-op Industrial Estate, Balanagar, Hyderabad - 500 037, India. E-mail - jagathreddy@usa.net; Fax \# 91-40-23773487.

\begin{abstract}
A number of 2-(1,3-Diarylpyrazol-5-ylamino)-4-(3-oxo-2H-1,4-benzoxa/thiazin6-yl)-thiazoles (5a-k \& 6a-d) have been prepared.
\end{abstract}

\section{Introduction}

The emergence of pyrazole based drugs like Celecoxib ${ }^{1}$ and Sildenafil citrate ${ }^{2}$ has created great interest in the synthesis of a variety of this class of compounds. Thiazole ring forms a part of a number of synthetic ${ }^{3}$ and natural products ${ }^{4}$ of medicinal interest. Several substituted aminopyrazoles and aminothiazoles are known to exhibit antimicrobial activities ${ }^{5}$. Furthermore, several substituted benzoxazines and benzothiazines have been reported as antihypertensive ${ }^{6}$, antiulcer ${ }^{7}$ and herbicidal agents ${ }^{8}$. In view of this and in continuation of our work on new 1,4-benzoxazines, we report herein the synthesis of 2-pyrazolylamino-4-benzoxa/thiazinyl thiazoles as possible antibacterial and antifungal agents.

\section{Results and Discussion}

The various aminopyrazoles (1) required in the present work were prepared by reaction of benzoylacetonitriles with phenylhydrazines in a modified reaction condition using montomorillonite $\mathrm{K} 10$ under acid free conditions ${ }^{10}$. The aminopyrazoles 1 were converted into the corresponding thioureas 2 by reaction of 1 with benzoylisothiocyanate followed by hydrolysis according to the reported methods ${ }^{11}$. 6-Chloroacetyl benzoxa/thiazinones $3 \& 4$ were prepared by chloroacetylation of benzoxa/thiazinones under Friedel-crafts reaction conditions. Reaction of $3 \& 4$ with 2 in refluxing ethanol gave the desired pyrazolylaminobenzoxa/thiazinyl thiazoles 5 \& 6 in good yields (Scheme -1). The structures of various $5 \& 6$ thus prepared were established based on their ${ }^{1} \mathrm{H}$ NMR and analytical data (Table -1 ). In the ${ }^{1} \mathrm{H}$ NMR spectra compounds 5 \& 6 exhibited typical singlets for $\mathrm{OCH}_{2}(\delta 4.56), \mathrm{SCH}_{2}(\delta 3.77)$, pyrazole (6.81), thiazole (6.84) and lactam $\mathrm{NH}(10.2-10.5)$ protons apart from aromatic protons.

\section{Experimental Section}

Melting points were determined in open capillaries and are uncorrected. IR spectra recorded in $\mathrm{KBr}$ pellets. ${ }^{1} \mathrm{H} \mathrm{NMR}$ spectra on a Varian $200 \mathrm{MHz}$ instrument with TMS as internal standard, chemicals shifts expressed in $\delta$ ppm and Mass spectra on Hewlett Packard Mass spectrometer operating at $70 \mathrm{eV}$. 


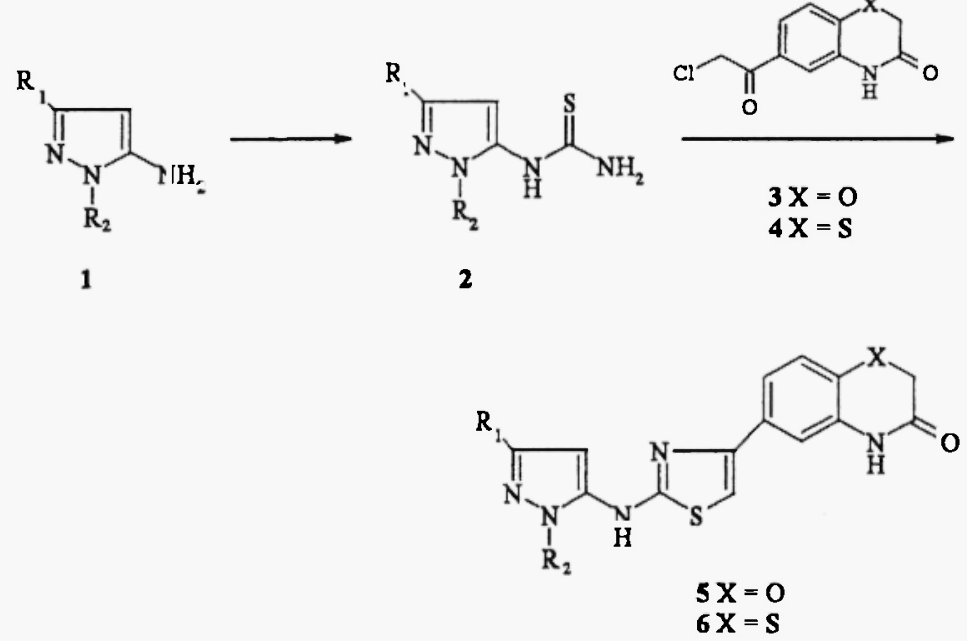

S C H E M E - 1

General procedure for the preparation 1-(1,3-diaryl-pyrazol-5-yl)thiourea 2

To a mixture of ammonium thiocyanate $(0.012$ mole) in acetone $(25 \mathrm{ml})$, benzoylchloride $(0.01$ mole) was added drop wise and the mixture was refluxed for 15 minutes. A solution of $1(0.01 \mathrm{~mole})$ in acetone $(25 \mathrm{ml})$ was added drop wise to the above reaction mixture, so that a gentle reflux was maintained. The separated solid was filtered and refluxed with $10 \%$ aq. $\mathrm{NaOH}(25 \mathrm{ml})$. It was filtered and the filtrate was acidified with Conc $\mathrm{HCl}$. The solid obtained was recrystallized from methanol to give pure 2 . The melting points and yields of different pyrazolyl thioureas prepared given below.

2a $\left(\mathrm{R}_{1}=4-\mathrm{CH}_{3} \mathrm{C}_{6} \mathrm{H}_{4}, \mathrm{R}_{2}=\mathrm{C}_{6} \mathrm{H}_{5}\right) 223^{\circ}, 82 \% ; 2 b\left(\mathrm{R}_{1}=4-\mathrm{ClC}_{6} \mathrm{H}_{4}, \mathrm{R}_{2}=\mathrm{C}_{6} \mathrm{H}_{5}\right) 209^{\circ}, 84 \%$; 2c $\left(\mathrm{R}_{1}=4-\mathrm{BrC}_{6} \mathrm{H}_{4}, \mathrm{R}_{2}=\mathrm{C}_{6} \mathrm{H}_{5}\right) 222^{\circ}, 83 \%$; $2 \mathrm{~d}\left(\mathrm{R}_{1}=4-\mathrm{FC}_{6} \mathrm{H}_{4}, \mathrm{R}_{2}=\mathrm{C}_{6} \mathrm{H}_{5}\right) 211^{\circ}, 86 \% ; 2 \mathrm{e}$ $\left(\mathrm{R}_{1}=4-\mathrm{CH}_{3} \mathrm{C}_{6} \mathrm{H}_{4}, \mathrm{R}_{2}=4-\mathrm{ClC}_{6} \mathrm{H}_{4}\right) 229^{\circ}, 81 \% ; 2 \mathrm{f}\left(\mathrm{R}_{1}=\mathrm{R}_{2}=4-\mathrm{ClC}_{6} \mathrm{H}_{4}\right) 189^{\circ}, 79 \% ; \mathbf{2 g}\left(\mathrm{R}_{1}\right.$ $\left.=4-\mathrm{BrC}_{6} \mathrm{H}_{4}, \mathrm{R}_{2}=4-\mathrm{ClC}_{6} \mathrm{H}_{4}\right) 171^{\circ}, 78 \% ; 2 \mathrm{~h}\left(\mathrm{R}_{1}=4-\mathrm{CH}_{3} \mathrm{C}_{6} \mathrm{H}_{4}, \mathrm{R}_{2}=2,4-\mathrm{diF}\right) 222^{\circ}, 79 \%$; $2 i\left(R_{1}=4-C_{1 C} \mathrm{H}_{4}, \mathrm{R}_{2}=2,4-\mathrm{diF}\right) 208^{\circ}, 84 \% ; 2 \mathrm{j}\left(\mathrm{R}_{1}=4-\mathrm{FC}_{6} \mathrm{H}_{4}, \mathrm{R}_{2}=2,4-\mathrm{diF}\right) 219^{\circ}, 87 \%$; 2k $\left(\mathrm{R}_{1}=4-\mathrm{BrC}_{6} \mathrm{H}_{4}, \mathrm{R}_{2}=2,4-\mathrm{diF}\right) 218^{\circ}, 79 \%$.

General procedure for the preparation of 2-pyrazolylamino-4-benzoxa/thiazin-6yl thiazoles $5 \& 6$.

A mixture of 2 ( $0.01 \mathrm{~mole})$ and 6-chloroacetyl benzoxa/thiazinone $(3$ or $4,0.01$ mole) in ethanol $(50 \mathrm{ml})$ was refluxed for $4-5 \mathrm{hrs}$. At the end of the reaction as monitored by TLC, the solvent was evaporated, the residue was neutralized with $\mathrm{NaHCO}_{3}$ solution and the resulting solid was filtered and recrystallized from methanol to give pure (5 \& 6).

2-(1-Phenyl-3-tolyl-pyrazol-5-yl)amino-4-(3-oxo-2H-1,4-benzothiazin-6-yl)-thiazole 6a $\left(\mathrm{R}_{1}=4-\mathrm{CH}_{3} \mathrm{C}_{6} \mathrm{H}_{4}, \mathrm{R}_{2}=\mathrm{C}_{6} \bar{H}_{5}\right)$

Pyrazolyl thiourea $2\left(\mathrm{R}_{1}=4-\mathrm{CH}_{3} \mathrm{C}_{6} \mathrm{H}_{4}, \mathrm{R}_{2}=\mathrm{C}_{6} \mathrm{H}_{5}\right)$ was converted into $6 \mathrm{a}$ according to the general procedure described above to give $\mathbf{6 a}$ as white crystalline solid $72 \%$, m.p $233^{\circ}$. ${ }^{\circ} \mathrm{H}$ NMR $\left(\mathrm{CDCl}_{3}+\mathrm{DMSO}_{6}, 200 \mathrm{MHz}\right): \delta 2.38(\mathrm{~s}, 3 \mathrm{H}), 3.37(\mathrm{~s}, 2 \mathrm{H})$, 6.81(s, 1H), 6.94(s, 1H), 7.19-7.82(m, 12H), 9.51(bs, 1H), 10.17(bs, 1H). Found: C, $69.63 ; \mathrm{H}, 4.67 ; \mathrm{N}, 15.26 \mathrm{C}_{27} \mathrm{H}_{21} \mathrm{~N}_{5} \mathrm{OS}_{2}$ requires $\mathrm{C}, 69.97, \mathrm{H}, 4.53 ; \mathrm{N}, 15.11 \%$. 


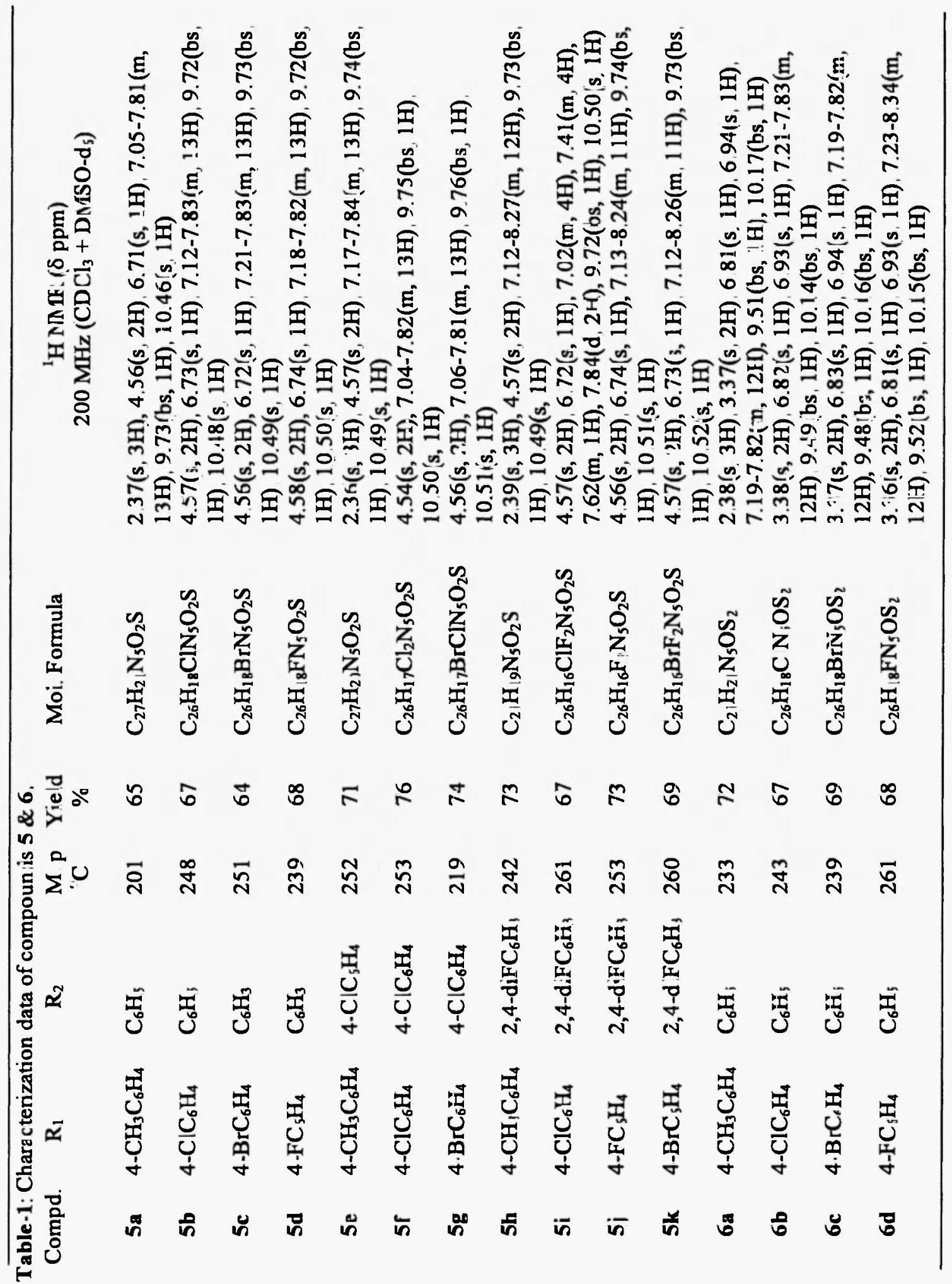




\section{References}

1. T. D. Penning, J. J. Talrey, S. R. Bertenshaw, J. S. Carter, P. W. Collins, S. Docter, M. J. Graneto, L. F. Lee, J. W. Malecha, J. M. Miyashiro, R. S. Rogers, D. J. Rogier, S. S. Yu, G. D. Anderson, E. G. Burton, J. N. Cogburn, S. A. Gregory, C. M. Koboldt, W. E. Perkins, K. Siebert, A. W. Veenhuizen, Y. Y. Zhang, P. C. Isakson, J. Med. Chem, 40, $1997,1347$.

2. A. Martel, A. Graw, X. Rabasseda, R. Castaner, Drugs of Future, 22, 1997, 138.

3. M. Chihiro, H. Nagamoto, I. Takanura, K. Kitano, H. Komatsu, K. Sekigudi, F. Tabusa, T. Mori, M. Tominaga, Y. Yabuuchi, J. Med. Chem, 38, 1995, 353.

4. H. Abe, T. Takaishi, T. Okuda, Tetrahedron Lett, 19, 1978, 2794.

5. a) J. Elguero in Comprehensive Heterocyclic Chemistry II edited by A. R. Katritzky, Pergamon Press (Oxford) 3, 1996, 67.

b) A. Dondoni, P. Merino in Comprehensive Heterocyclic Chemistry II, edited by A. R. Katritzky, Pergamon press (Oxford), 3, 1996, 465.

6. C. B. Chapleo, R. C. M. Batler, D. England, P. L. Myen, A. G. Runach, C. F. C. Smith, M. R. Stiuings, I. F. Talloch, J. Med. Chem, 32, 1989, 1627.

7. Y. Katsura, S. Nishiro, H. Takasugi, Chem. Pharm. Bull, 39, 1991, 2937.

8. O. Schallner, H. G. Schwarz, D. Hoischen, K. H. Linker, M. W. Drewes, F. P. Danman, D. P. Fenchi, R. Pontzers, W. O 0206, 277; Chem. Abstr; 136, 2002, $118455 \mathrm{~g}$

9. a) Jagath Reddy, S. Sailaja, K. Srinivasa Rao, P. Reddanna, D. Bharat Reddy, Indian J. Chem, 2004 (in press)

b) P. S. N. Reddy, Pragati Reddy, G. Jagath Reddy, K. Srinivasa Rao, Heterocyclic Commun., 9, 2003, 503.

10. G. Jagath Reddy, D. Latha, K. Srinivasa Rao, Org. Pren. Proced. Intl, 2004 (in press).

11. M. R. H. Elmoguayar, M. K. A. Ibrahim, F. M. Darwish, Org. Prepn. Proced. Intl., $16,1984,1$.

\section{Received on June 18, 2004}

